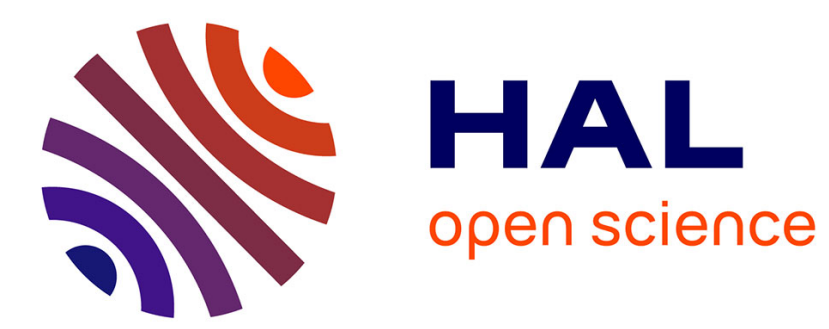

\title{
Ces bonnes vaches aux yeux si doux
}

André Micoud

\section{- To cite this version:}

André Micoud. Ces bonnes vaches aux yeux si doux. Communications, 2003, 74 "Bienfaisante nature", pp.217-237. hal-00151426

\section{HAL Id: hal-00151426 \\ https://hal.science/hal-00151426}

Submitted on 4 Jun 2007

HAL is a multi-disciplinary open access archive for the deposit and dissemination of scientific research documents, whether they are published or not. The documents may come from teaching and research institutions in France or abroad, or from public or private research centers.
L'archive ouverte pluridisciplinaire HAL, est destinée au dépôt et à la diffusion de documents scientifiques de niveau recherche, publiés ou non, émanant des établissements d'enseignement et de recherche français ou étrangers, des laboratoires publics ou privés. 
André Micoud

Ces bonnes vaches aux yeux si doux

\section{Avertissement.}

La vache, sous sa forme folle, est venue tout à coup envahir notre actualité. Et c'est cette irruption qui nous a fait reprendre ici un ancien projet d'écriture resté depuis plusieurs années dans des cartons, et où nous nous posions la question : mais qu'est-il donc arrivé aux animaux industrialisés ? La réponse vient d'être donnée : quelques-uns deviennent fous. Jusqu'à ce que, peut-être, certains d'entre nous, atteints à leur tour par la maladie de Creutzfeld-Jakob, les suivent dans cette déraison. A moins que cette déraison, d'une autre manière, ait commencé depuis longtemps déjà.

Reprenant le dossier pour une revue qui s'intéresse à la communication, il nous est apparu que, de ce point de vue, on pouvait distinguer trois sortes de vaches.

\section{Où il est question de trois catégories de vaches.}

La première catégorie concerne les vaches qui, bien qu'invisibles aux yeux de chair, sont innombrables en ce qu'elles peuplent les souvenirs nostalgiques des plus que quinquagénaires. Elles s'appelaient Margot ou Fleurette et leurs yeux étaient infiniment doux. Elles étaient celles du grand-père auprès de qui on allait passer des vacances et dont, le soir à l'étable, on buvait le lait bourru qui écume dans les seaux. À peine sont-elles évoquées, que l'envie vient de les faire revivre. Burlesques, quand elles devaient déguerpir à travers prés en jetant vers le ciel leur arrière-train disgracieux. Placides, quand leurs ruminations pouvaient inspirer des méditations métaphysiques interminables. Impudiques, quand leurs bouses s'esclaffaient dans la cour de la ferme ou quand leurs chevauchements contre-nature intriguaient les petits enfants curieux et craintifs. Et tendant leurs museaux baveux comme pour quêter un baiser impossible... On pourrait, dans cette veine, aligner les textes fort nombreux d'auteurs des générations passées qui se sont essayé à ces efforts de reviviscence. On en trouve de beaux extraits dans l'Anthologie des textes littéraires sur la vache ${ }^{1}$. Qu'il nous soit permis de citer seulement les quelques lignes par lesquelles Georges Picard, dans Le vagabond approximatif commence un court récit consacré aux vaches du Berry :

\footnotetext{
1 Pascal Commère, Paris, éd. P.M Favre, 1998.
} 
"On trouve ce que l'on veut dans le regard des vaches. C'est sans doute pour cette raison qu'elles suscitent la sympathie humaine. Leurs gros yeux sont des miroirs de compréhension et d'affection. Les vaches acceptent et pardonnent tout, écoutent les confidences, ménagent notre susceptibilité et, telles de plantureuses matrones pour lesquelles la vie est sans complications psychologiques ni détours existentiels, elles n'ont que leur chaleur animale et leur bon sens champêtre à échanger"'2.

Toutefois, aussi nombreuses qu'elles puissent être, les vaches de cette première catégorie resteront toujours secrètes. Leur existence n'est que celle de l'intimité qui a su les accueillir et qui en garde le souvenir.

Tel n'est pas le cas de la seconde catégorie de vaches : les vaches en images. Celles-là sont bien visibles. Leurs représentations envahissent de plus en plus les campagnes... de promotion, non seulement des fromages et du lait, des yaourts naturels et autres produits fermiers, mais des terroirs authentiques, des qualités de la profession d'éleveur, de l'honnêteté des grands distributeurs... Beaucoup d'observateurs l'ont déjà remarqué : la vache, en image, est de plus en plus présente, partout sur les murs des villes ou dans les magazines.

Dans ces apparitions d'un nouveau genre, un œil plus exercé pourrait cependant distinguer deux sous-catégories. La première, toujours sur fond de nostalgie, représente à satiété pourrait-on dire la vache mythique ou éternelle. Image de la tranquillité rassurante et de l'abondance toujours renouvelée, elle est là pour combler l'enfant goulu de son pis généreux. L'accompagne souvent la crémière accorte qui, quelques mollettes de beurre dans son panier ou une biche à lait au bras, gambade vers la laiterie. La maternité, la prime enfance, la jeunesse insouciante, tels sont les ingrédients que l'on trouve de façon récurrente sur les étiquettes commerciales. Toujours dans cette première sous-catégorie, "passéiste" pourrait-on dire, est venue s'ajouter récemment la vache "naturelle". Celle des alpages par exemple qui, la tête encore ornée de cornes, se détache majestueusement sur fond de montagnes enneigées ; comme si elle se savait déjà forte de sa nouvelle légitimité écologiquement correcte, et touristiquement attirante ${ }^{3}$.

Plus énigmatique est la seconde sous-catégorie de cette vache proliférante en image : la vache artistique. Tour à tour surréaliste, cubiste, dadaïste ou psychédélique, affublée de tous les attributs possibles et imaginables, c'est la vache de l'imaginaire débridé des jeunes créateurs; depuis Dubuffet (années 50), dont Alexandre Vialatte a décrit les créatures ("Vaches mauves" et "Chronique de la vache étonnée") ${ }^{4}$ jusqu'à la "Miss Helvetia" de Niki de Saint Phalle, en passant par la pochette de disque des Pink Floyd (années 70). Comme si sa placidité proverbiale la rendait infiniment disponible aux multiples métamorphoses que lui font subir les

\footnotetext{
2 Paris, José Corti, 2001. Tous mes remerciements à Bernadette Lizet à qui je dois cette trouvaille.

${ }^{3}$ Voir le numéro 11 de la revue Alpes consacré à "L'or des alpages. Fromages et fromagers", avril-juin 2001. Sur cette "vache des montagne", on pourra lire aussi le $\mathrm{n}^{\circ} 4$, tome 86,1998 , de la Revue de Géographie Alpine (sur le thème "Patrimoine, montagne et biodiversité") dirigé par Laurence Bérard et Philippe Marchenay.

${ }^{4}$ Alexandre Vialatte, Jean Dubuffet et le Grand Magma, Arléa, 1988. Que Cécile Tardy, à qui je dois cette précieuse référence, soit ici remerciée.
} 
artistes plasticiens ${ }^{5}$. On trouvera un florilège de ces "vaches folles" dans le magnifique ouvrage Nos vaches ${ }^{6}$, publié avec l'aide du CIDIL $^{7}$ par une maison d'édition associative, dont le nom est tout un programme. Tout le monde connaît la première de cette série d'images, intermédiaire entre nos deux sous-catégories: "La vache qui rit", due au talent de Benjamin Rabier qui, après avoir illustré la boîte du camembert Saint-Hubert produit en Lorraine dans les années 30, a connu un succès foudroyant quand elle passa chez $\mathrm{Bel}^{8}$. En bref, et comme l'écrivait plus récemment Arte Magazine en annonce de sa soirée thématique consacrée à la vache ("Drôlement vache, vaches folles et vaches sacrées", dimanche 23 janvier 1999): "Après le veau d'or, la vache culte : le lymphatique ruminant de nos pâturages atteint aujourd'hui des sommets de popularité...".

Vient enfin la troisième catégorie de vaches, bien réelles celles-là, mais devenues presque invisibles et qui le seraient sans doute restées si l'encéphalite spongiforme bovine n'en avait révélé l'existence aux téléspectateurs ahuris. C'est à ces dernières que cet article est dédié. Il commencera par une description la plus objective possible - comme il se doit pour un être vivant qui est devenu un quasiobjet - de la vie ordinaire d'une de ces vaches ordinaires. Tel nous a semblé le bien qu'il était possible de leur faire : révéler leurs conditions d'existence aux lecteurs, sans doute très nombreux, qui en ignorent à peu près tout ${ }^{9}$. Il nous sera possible alors, avec l'aide du philosophe de la technique, Gilbert Simondon ${ }^{10}$, de nous demander comment parler du mode d'existence de ces êtres que le terme de "domestique" ne peut plus servir à définir.

\section{Description de la vie ordinaire d'une vache standard actuelle.}

\footnotetext{
${ }^{5}$ Voir Vache d'expo, catalogue de l'exposition à la Ménagerie de Verre, Paris 1992, publié par Un sourire de toi..., La vache et l'homme, catalogue d'une exposition réalisée au musée de Caen, et encore la vache de Pol Bury qui faisait partie de l'exposition itinérante Dessine-moi une vache et qui a été acquise récemment par le musée de Dijon (donation Grandville). Un site du même intitulé rassemble un grand nombre d'artistes et organise un concours permanent. (www.pilotmotiv.2svi.com/dessinemoivachebis.htm); on y trouvera un grand nombre d'autres liens. Une artiste peintre stéphanoise, Annick Claude, a développé ainsi toute une partie de son œuvre graphique autour de la vache (Musée d'Art Moderne de Saint-Etienne).

${ }^{6}$ Paris, Un sourire de toi et j'quitte ma mère, 1998.

${ }^{7}$ Centre d'Information et de Documentation de l'Industrie Laitière. On notera que, significativement, le développement de ce sigle ne figure nulle part dans l'ouvrage.

${ }^{8}$ En 1921, Léon Bel, jeune entrepreneur de l'industrie fromagère, réalisa un croquis d'une vache pour son "fromage moderne", inspiré de celui que Benjamin Rabier avait fait pour la section de ravitaillement à laquelle ils avaient été affectés tous les deux pendant la guerre de 14-18 (et qu'il avait déjà vendu à d'autres fromagers). Non satisfait de son coup de crayon, Léon Bel fît appel au talent de son ancien camarade. Avec ses fameuses boucles en abîme, le nouveau dessin est l'ancêtre de celui qu'on connaît aujourd'hui. Voir Vincent Vidal et Guillaume Villemot, La chevauchée de la vache qui rit, Paris, éd. Hoebeke Lionel, 1991.

${ }^{9}$ Je voudrais ici remercier tout particulièrement Gilbert Nicaise, technicien agricole à la retraite, qui a bien voulu me faire partager sa longue expérience. Mes remerciements vont aussi à Philippe Pluvinage et Marc Delacroix qui m'ont fait part de leur avis sur une première version de ce texte.

${ }^{10} \mathrm{Du}$ mode d'existence des objets techniques, Paris, Aubier, 1989.
} 
Quelques précisions liminaires s'imposent. La vache dont il va être question n'existe pas autrement que dans et par cette description. Il s'agit donc, comme le dit le langage sociologique, d'une vache idéal-typique. "Pour ne pas ennuyer le lecteur avec des statistiques, on considérera que la description concerne la vie de la très grande majorité des vaches laitières élevées dans des troupeaux de taille moyenne, appartenant à des "exploitations modernes" françaises, soit près de $80 \%$ des vaches existant réellement sur le territoire. Dans ces conditions, on ne tiendra compte ni des particularités régionales, ni des différences entre les races, ni des modalités singulières qui font que telle ou telle pratique d'élevage est déjà répandue ici quand elle n'est encore qu'en développement ailleurs. Cette description suit "naturellement" le cycle de vie d'une vache, depuis sa naissance jusqu'à sa mort.

\section{Où l'on s'aperçoit que, pas encore née, une vache est déjà programmée...}

Pour une vache en effet, le cours naturel d'un récit de vie ne commence pas quand elle est mise au monde en tant que veau femelle. Faire l'histoire de vie d'une vache implique que soient prises en compte les connaissances scientifiques qui président à sa conception et qui concernent sa génitrice présumée. Soit donc une vache laitière dont le potentiel génétique productif est connu11, et à qui l'éleveur a décidé de faire faire un veau. Les caractéristiques de la vache choisie pour être mère ont été comparées avec celles de la moyenne de l'étable et de la moyenne de sa race. Une série d'indices se dessinent (matière grasse, matière protéique...), qui vont commander le type de taureau avec lequel il est préférable de l'accoupler. Les caractéristiques de ce reproducteur ont déjà été testées pendant trois ans à partir de sa descendance femelle (généralement sur un échantillon d'une centaine de vaches laitières dont on mesure la production des cent premiers jours de lactation). Le taureau est choisi de telle sorte qu'il vienne encore améliorer les potentialités productives de la génitrice ou permettre de remédier à certaines de ses insuffisances, notamment de la qualité de son lait. Quelques mots au passage sur le sort de ce taureau, issu lui aussi d'accouplements programmés. Bien entendu, il ne se déplace pas pour accomplir son œuvre fécondatrice; c'est l'inséminateur qui vient dans sa camionnette avec ses petites paillettes pour faire le travail. Ou bien les qualités du taureau sont reconnues : il donne sa semence pendant quatre ou cinq ans (congelée dans l'azote liquide), et passe ces jours dans un Centre Régional d'Insémination Artificielle, organisme professionnel ayant en charge la sélection et l'amélioration des races. Ou bien il est castré et engraissé en tant que bœuf pour la filière viande.

La vache a été inséminée ${ }^{12}$. Neuf mois plus tard vient donc le vêlage et le début de l'histoire de vie de l'être qui nous intéresse : un veau femelle.

\footnotetext{
${ }^{11}$ Ceci grâce au Contrôle Laitier. Le premier syndicat de contrôle laitier fut créé en 1907 au Pays de Caux en Seine Maritime. Cet organisme professionnel tient le registre de toutes les vaches pour lesquelles il est sollicité.

${ }^{12}$ Avec un taux de réussite moyen à la première insémination inférieur à celui qui est fixé comme objectif $(65 \%)$.
} 
Dès qu'elle est née, il s'agit de la préparer à produire

On désinfecte le cordon ombilical. On laisse la mère sécher son veau ou on le sèche à sa place. Parfois, dès la naissance, et en cas de besoin, on donne un peu de vitamines. Mais l'important est de s'assurer que le veau tête le colostrum, au moins deux fois. Après quoi, on le sépare de sa mère et on lui donne à boire au seau avec tétine, soit du lait de vache reconstitué, soit le lait de sa mère ou celui du troupeau (de plus en plus fréquemment depuis la politique des quotas : en note, pourquoi ?),. Dans la pratique ancienne (jusqu'aux années 70 ) le veau tétait sa mère de quinze jours à trois semaines, avec des tétées rationnées puisqu'il lui était impossible d'absorber tout le lait de sa mère. Cette pratique n'a quasiment plus cours aujourd'hui car elle endommageait les trayons et le sevrage était stressant. Au bout $\mathrm{du}$ huitième jour sont introduits progressivement les "starters", aliments de complément. Additionnés au lait sous forme de flocons, ils ont pour but de mettre en route le système de digestion. On donne aussi à discrétion du "petit foin" à fibre fine avec de l'eau claire, afin d'habituer le veau à manger et, surtout, pour favoriser immédiatement le développement de la panse. Le lait est diminué au fur et à mesure que sont augmentées les rations d'aliments starter et de foin. C'est ce régime alimentaire que les veaux femelles vont connaître pendant six mois. Ils logent pendant deux à trois semaines dans des niches en plastique (un peu plus spacieuses que des niches à chien), devant lesquelles sont disposés les aliments. Puis on met les futures génisses dans des petits parcs en été, ou dans un coin à part du bâtiment de stabulation pendant l'hiver.

Signalons également que deux à trois semaines après la naissance, les bourgeons des cornes sont brûlés à l'aide d'un appareil électrique. Cette pratique s'est généralisée afin d'éviter les blessures consécutives aux bousculades entre animaux et dans le but de protéger les éleveurs contre les accidents. Une autre raison, moins souvent évoquée par les techniciens, tient sans doute au fait que des vaches sans cornes peuvent plus facilement accéder aux boxes individualisés de l'auge collective équipés de systèmes de barreaux mobiles.

\section{Où les futures vaches connaissent quelques moments de liberté}

Six mois après leur naissance, les génisses sont placées dans un plus grand parc - une prairie artificielle pas trop éloignée de l'exploitation - où elles commencent à pâturer. Elles sont ornées à l'oreille d'une boucle d'identification, parure obligatoire dès la naissance, généralement de couleur jaune, dont le numéro est déposé à l'Établissement Départemental d'Élevage (organisme professionnel institué par décret). Ce numéro, quasi-équivalent de celui de la Sécurité Sociale, permet de connaître individuellement toutes les bêtes. Il est composé du numéro de code du département, de celui de l'exploitation et du rang de naissance de l'animal dans l'exploitation. S'y ajoute une lettre qui change chaque année, et qui peut servir encore d'initiale au "petit nom" que l'éleveur donnera à son animal ${ }^{13}$. Pour parer au

\footnotetext{
${ }^{13}$ Les avis des différentes personnes consultées divergent sur ce point : si toutes s'accordent à dire que les génisses sont encore nommées, il est difficile, faute d'enquêtes en profondeur, d'en savoir plus quant à l'usage effectif de ces noms par les éleveurs. Sur ce sujet on pourra se reporter à l'article ainsi
} 
cas de la perte accidentelle de la boucle, le Contrôle Laitier procède également à un tatouage. Ainsi répertoriées, les génisses demeurent dans leur parc pendant un an à pâturer, à folâtrer peut-être, mais aussi à se nourrir de "granulé deuxième âge", de céréales aplaties, de foin sec, de compléments de fourrage (du foin, parfois remplacé en hiver par de l'ensilage, herbe ou maïs fermenté). Des minéraux sont également apportés. C'est pendant cette période qu'elles auront parfois à subir les traitements curatifs contre les maladies des jeunes (coccidiose, entérites) et qu'elles pourront être vaccinées contre la pneumonie.

\section{Génitrices puis vaches laitières}

À dix-huit mois, vient le moment où l'on surveille l'apparition des premières chaleurs repérables à la congestion de la vulve, aux épanchements de glaires ainsi qu'aux agitations (chevauchements). On choisit généralement la fécondation "en race pure", pour engendrer une génisse destinée à devenir à son tour une vache laitière. On évite ainsi les problèmes de vêlage provoqués par une "I.A. » (insémination artificielle) en croisement ${ }^{14}$. La jeune vache reçoit une "ration de gestation" calculée à partir de la quantité de fourrage qu'elle peut ingérer et composée d'habitude d'un mélange de tourteaux, de céréales et de minéraux. Cette alimentation très poussée est destinée à assurer la fin de croissance de la future mère, la constitution de ses réserves, le développement du fœtus et la lactation à venir. Ainsi, dès la naissance de son veau, elle est en mesure de produire du lait en abondance en consommant ses réserves. Quinze jours avant le vêlage, elle change de ration, dite alors "de préparation à la lactation", comportant une plus forte teneur en azote de manière à obtenir le plus rapidement possible le pic de la production laitière. Dans le même temps, on soumet la vache aux tests de contrôle contre la tuberculose (la périodicité sera ensuite de un à trois ans selon la situation épidémiologique de la zone). Avant sa mise au troupeau, on vérifie par ailleurs qu'elle n'est pas porteuse de maladies contagieuses, telles que la brucellose, l'IBR (rhino-tracéite infectieuse bovine), ou la leucose. Enfin, immédiatement après le vêlage, une nouvelle "ration de production" lui est fournie, calculée à partir de sa production escomptée d'après les potentiels génétiques de ses géniteurs. Dans le cas général, cet objectif théorique est cependant difficile à atteindre. La vache maigrit - ce qui est physiologiquement normal - et l'on ne parvient pas à lui faire manger tout ce qu'elle devrait théoriquement ingurgiter. Pour lui faire retrouver son appétit, on peut recourir à des injections de stimulants de la sécrétion hépatique (afin d'éviter la crise de foie) et aussi parfois, surtout en hiver, quelques vitamines.

La vache laitière en troupeau

qu'à la bibliographie très complète de Jocelyne Porcher, "La relation de communication entre l'éleveur et ses animaux : un domaine encore à explorer", dans Le Courrier de l'environnement de l'INRA, $\mathrm{n}^{\circ} 32$, 1997, pp. 51-62.

${ }^{14}$ En croisement, un taureau appartenant à une race à viande donne un veau à musculature plus forte à la naissance. 
Quinze jours après la mise bas, la jeune vache est introduite dans le troupeau des laitières. L'éleveur doit pour cela user de quelques astuces pour éviter le stress, un stress normal pour un animal qui vient de faire l'expérience d'un vêlage, et se retrouve d'un coup dans un grand troupeau où il doit apprendre à faire sa place. C'est également pour éviter le stress consécutif au sevrage que le veau lui a été enlevé dès la naissance et qu'elle est traitée avec toutes les autres laitières du troupeau. Un autre objectif est en jeu : lui faire atteindre le plus rapidement possible son pic de production de lait, au bout d'un mois à un mois et demi, en moyenne. Restera alors à empêcher que ce volume de lait quotidien ne descende trop rapidement. Après cent jours de traite, grâce à la pesée mensuelle de son lait par le Contrôle Laitier, on sait quelle sera sa production totale. Sa ration d'aliments de complément est calculée en fonction de cette production, par rapport aux moyennes de l'étable et à celles de la race. La bête porte au cou un collier électronique dans lequel sa ration est programmée; elle peut s'alimenter aux différents silos qui lui délivrent exactement la quantité prescrite. Précisons quand même, pour éviter tout malentendu, que l'herbe reste le premier et le plus important des aliments de la vache. Pendant l'été, elle pâture le plus souvent dans les prairies artificielles; pendant l'hiver, elle ingère l'herbe sous forme de foin, avec ou sans ensilage (herbe et maïs). Les tourteaux, les minéraux et les céréales n'interviennent qu'en faible volume. Toutefois, c'est bien dans ces aliments concentrés qu'interviennent les matières riches en protéines (MRP) si importantes lors de la lactation, et dont les fameuses farines animales ${ }^{15}$ ont fait partie.

Quand elle n'est pas dans les prés, l'existence au jour le jour de la jeune laitière est alors rythmée par les traites bi-quotidiennes et par les apports de fourrage et d'ensilage, de plus en plus souvent effectués par remorques auto-distribuantes approvisionnant l'auge collective. Si elle fait partie d'une exploitation qui pratique le "zéro pâturage" (apport sur place d'herbe coupée le jour même dans les prairies), elle passera ses journées avec ses congénères à déambuler dans un enclos constitué d'une partie à l'air libre et d'une partie couverte : c'est la stabulation «libre ${ }^{16}$. Les déjections sont ramassées régulièrement par divers moyens mécaniques et constituent, mélangées à la paille de la litière, la fumure pour les cultures. La salle de traite qui jouxte le grand bâtiment de la stabulation libre est très propre. La machine à traire peut selon les cas débiter de trois à vingt vaches à la fois, avec des systèmes variés de tourniquets empêchant les animaux de se bousculer. Le lait passe directement du pis au tank réfrigéré, que le camion citerne du ramassage vient

\footnotetext{
${ }^{15}$ Pour les vaches laitières, ces farines animales représentaient moins de $1 \%$ de la ration. Sur ce sujet à haut risque, on pourra consulter le rapport de Jean Bizet et Gérard Deriot (www/senat.fr).

${ }^{16}$ Fortement encouragée par la profession dans les années 70 au titre de l'intensification, la technique du "zéro pâturage" (zero grassing) n'a pas été suivie partout et serait actuellement en régression. Les seules à l'avoir conservée, à cause des difficultés de déplacement des troupeaux, sont les exploitations proches des agglomérations, ou celles dont la structure foncière est éclatée. Notre vache standard passe donc environ quatre mois, entre le printemps et l'été, à brouter dans les prés. Les techniciens interrogés, qui ont tous à cœur de donner une image estimable des éleveurs - surtout s'adressant à un "urbain"-, insistent sur le fait que la stabulation libre (vue parfois comme un camp de concentration à vie par les urbains) constitue une amélioration certaine au regard des conditions d'hygiène et de mobilité réduite auxquelles étaient assujetties les vaches entravées pendant les longs mois d'hiver, dans des étables obscures et souvent mal nettoyées.
} 
aspirer régulièrement. Quand la production de notre vache a trop baissé (au bout d'un peu plus de 300 jours), vient alors le temps du tarissement. On sort la vache du lot des laitières et on réduit les apports de complément ; en été on la mettra sur une pâture de faible qualité. Le plus important à ce moment-là est d'éviter les mammites très contagieuses. C'est le moment que l'on choisit pour injecter des antibiotiques dans les trayons, pour ne pas prendre le risque de contaminer le lait destiné à la consommation humaine.

Sachant que l'intervalle moyen entre deux vêlages est de 380 jours (l'âge moyen au premier vêlage, en baisse constante depuis des années, étant actuellement de 31,7 mois), la vache peut maintenant entamer la deuxième phase de son cycle productif. Pendant ses deux à trois mois de tarissement, elle est moins nourrie, puisqu'il faut absolument éviter qu'elle se mette à engraisser. Puis, ce sera une nouvelle fécondation et le cycle reprendra. Comme tout son potentiel génétique est connu, c'est en fonction de celui-ci qu'on déterminera le reproducteur le plus apte à lui faire mettre au monde les veaux conformes. Ce cycle complet ne se reproduit que trois et quatre fois. Voilà une cinquantaine d'année une vache moyenne connaissait entre sept et huit périodes de lactation dans sa vie. Pourquoi cette diminution de la durée de vie moyenne des vaches? Les raisons en sont, par ordre d'importance : les problèmes de stérilité, les mammites, les boiteries et les troubles métaboliques. «Les vaches actuelles", nous dit un vétérinaire, "sont comme des Formules Un, très performantes, mais beaucoup plus fragiles et beaucoup plus difficiles à régler qu'une $2 \mathrm{cv}$ ». Le développement des acidoses, par exemple, tient au fait que les vaches ruminent de moins en moins, et produisent donc moins de salive ${ }^{17}$. On incrimine aussi les ensilages, équivalent de notre choucroute, ou plutôt les ensilages mal contrôlés contenant trop de corps cétoniques ${ }^{18}$.

La laitière devient alors vache de réforme. Elle change de genre grammatical pour devenir de la «viande de bœuf ». Rien de nouveau à cela : selon cette appellation bouchère, on appelle bœuf tout animal bovin de plus de huit mois. On fait alors prendre du poids à la vache et l'on se met en rapport avec la SICA d'élevage ${ }^{19}$ ou avec un maquignon qui viendra l'acheter au poids de la carcasse et au cours du jour. Pour savoir ce qui lui arrive ensuite, le lecteur pourra se reporter au beau livre que Noëlie Vialles a consacré aux abattoirs"l. Il y est montré, entre autres, comment l'abattage et la découpe transforment symboliquement le corps d'un animal vivant en une viande comestible par les humains. Resterait encore à dire ce qu'il advient de ses os et des autres parties de son organisme. Ou encore de sa peau. Si elle n'a pas été perforée par le varron (larve de la mouche Hypoderma bovis ou Hypoderma lineatum:), elle sera transformée en cuir pour fabriquer nos chaussures.

Mais il est temps de dire adieu à notre vache. Non pas parce que la description "objective" de sa vie serait terminée, mais parce qu'un tel pari de description objective confine à l'absurdité. La description de "la" vache ne peut pas être circonscrite à celle de son environnement immédiat. L'objectivité de ce récit est celle que nous avons

\footnotetext{
17 Les 300 grammes quotidiens de bicarbonate de soude que les marchands de produits phytosanitaires proposent de faire ingérer à l'animal pour remédier à cette carence sont peu de chose au regard des cinq kilos produits par la salive d'une seule vache (tu es sûr de ces chiffres ?).

${ }^{18}$ Dérivés carbonyles à double radical hydrocarboné.

${ }^{19}$ SICA : Société d'Intérêt Collectif Agricole.
} 
construite à partir des informations données par des exploitants ou des techniciens pour lesquels cette vache est objet de travail. Mais qu'advient-il du varron, hôte indésirable de notre animal ? Pour les techniciens et les éleveurs dont nous venons de parler, le varron est empoisonné à l'ivermectine et l'on n'en parle plus. Ils comparent cette campagne d'éradication à celles qui ont permis d'éliminer d'autres insectes ravageurs et ne trouvent rien à y redire. Mais il y a d'autres raisonnements. Le poison injecté à la vache se retrouve dans ses bouses, sans que le pauvre bousier et les autres insectes coprophages en aient été informés, ni les oiseaux qui en sont friands. De place en place, le système technique dont la vache constitue un des éléments finit par produire des effets bien au-delà de ce qui était imaginable. Jusqu'à, par exemple, produire des "printemps silencieux"20.

\section{La vache, un être vivant technicisé ?}

L'exemple du varron et celui de l'encéphalite spongiforme bovine le montrent à l'envi : obligation est faite aujourd'hui au chercheur de définir les limites de sa description. Ainsi, dans la description de la vie ordinaire d'une vache ordinaire, n'avons-nous jamais parlé de tous les coûts qu'impliquent la production et le maintien de cette vie, ni des emprunts, ni des crédits. De même, au motif apparemment fort raisonnable de ne pas allonger démesurément ce texte, nous n'avons pas jugé nécessaire de décrire dans le détail la multiplicité extraordinaire des organismes publics ou professionnels avec lesquels les éleveurs sont en contact permanent. Nous n'avons fait mention non plus des recherches zootechniques, biologiques, génétiques ou vétérinaires qui interviennent en amont de la filière. Nous n'avons pas fait état de tout ce qui concerne le machinisme agricole adapté à ces nouvelles manières de production, les techniques d'analyse des laits, la conduite des cultures de fourrage, etc. Comme Michel Callon a su le montrer avec ses mémorables coquilles Saint-Jacques de la baie de Saint-Brieux, le réseau socio-technique grâce auquel notre vache peut vivre est virtuellement extensible à la totalité de la société21.

Vient le moment de se poser une double question. Privée de ce réseau sociotechnique, la vache peut-elle vivre au-delà de quelques jours ou de quelques semaines ? Insérée dans un tel dispositif, peut-elle être encore dite "domestique" ? Pour répondre à ces deux questions, faisons un bref détour par l'œuvre de Gilbert Simondon ${ }^{22}$. Viendra ensuite le moment de poser une troisième question: quel rapport établir entre le réseau socio-technique et la prolifération des vaches en images à destination des urbains?

\footnotetext{
${ }^{20}$ D'après le titre de l'ouvrage célèbre d'une des pionnières de l'alerte écologique contre les pesticides, Rachel Carson, ancienne élève du limnologiste américain G. Evelyn Hutchinson (The silent spring, Paris, Plon, 1968, 2ème édition).

${ }^{21}$ Michel Callon, La science et ses réseaux. Genèse et circulation des faits scientifiques, Paris, La Découverte, 1989.

${ }^{22}$ Ouvrage cité.
} 
Les objets techniques selon Gilbert Simondon

L'auteur en distingue deux types: abstraits et concrets. Un objet technique abstrait est "la traduction physique d'un système intellectuel". Au contraire, "l'objet technique concret, c'est-à-dire évolué, se rapproche du mode d'existence des objets naturels... (puisqu'il) incorpore une partie du monde naturel qui intervient comme condition de fonctionnement"23. Ainsi pourrait-on dire d'une souris transgénique utilisée en laboratoire à des fins de recherches qu'elle est un "vivant construit abstrait", tandis qu'une plante transformée génétiquement et cultivée en pleine nature en est un qui "tend vers la concrétude"24. Gilbert Simondon propose une nouvelle définition de l'artificialité :

L'artificialité essentielle d'un objet réside dans le fait que l'homme doit intervenir pour maintenir cet objet dans l'existence en le protégeant contre le monde naturel, en lui donnant un statut à part d'existence. L'artificialité n'est pas une caractéristique dénotant l'origine fabriquée de l'objet par opposition à la spontanéité productrice de la nature: l'artificialité est ce qui est intérieur à l'action artificialisante de l'homme, que cette action intervienne sur un objet naturel ou sur un objet entièrement fabriqué... ${ }^{25}$

Exit la différence entre êtres vivants et objets fabriqués. La plante artificialisée «ne peut exister que dans ce laboratoire pour végétaux qu'est une serre ${ }^{26}$. "L'animal vivant technicisé" est lui aussi élevé aujourd'hui dans des "laboratoires", et il tend à devenir l'équivalent de la plante artificialisée. En effet, à mesure que celle-ci se développe, ce n'est bientôt plus que grâce à l'intervention artificialisante de l'homme, que l'animal "domestique » peut vivre. Les zootechniciens, quand ils ne considèrent pas l'animal comme "une machine thermodynamique dotée de mécanismes d'autorégulation"27, parlent à son sujet "d'animal domestique semi-libre ou confiné". Ce confinement des bêtes dans l'élevage moderne correspond à celui de la plante qui ne peut vivre qu'en serre ${ }^{28}$. Du coup, l'essentiel du problème se trouve être reporté peu à peu vers les conditions de possibilité de l'existence de l'objet technique, c'est-à-dire vers les diverses manières de "maintenir cet objet dans l'existence en le protégeant du monde naturel pour lui conférer un statut à part d'existence". "L'invention concrétisante », écrit encore Gilbert Simondon, « réalise un

\footnotetext{
${ }^{23}$ Ibidem, p. 46.

${ }^{24}$ Voir André Micoud, "Des objets vivant construits ?" dans Autour de l'œuvre de G. Simondon, à paraître aux Edition Universitaires de Saint-Etienne.

25 Souligné par nous.

26 Simondon, ouvrage cité, p. 47.

27 Etienne Landais et Joseph Bonnemaire, "La zootechnie, art ou science ?", dans Le Courrier de l'environnement de l'INRA, 27, 1996, pp. 23-44.

28 - C'est ici la vraie nouveauté des OGM : à savoir d'être des vivants artificialisés cultivés en pleine nature sans réel contrôle des effets interactifs entre eux et leur milieu.
} 
milieu associé techno-géographique qui est une condition de possibilité du fonctionnement de l'objet technique"29.

Résumons-nous et répondons en même temps aux deux questions que nous nous posions plus haut. L'objet technique le plus concret, c'est-à-dire celui qui est le plus capable de fonctionner presque tout seul ne le peut cependant que par son insertion dans un "milieu associé", qui fait couple avec lui, et dont l'entretien exige l'intervention continue de l'homme. Ce milieu associé, c'était hier ce qu'on appelait l'élevage domestique. Il a peu à peu généré un animal qu'il n'est plus possible de continuer à désigner comme «domestique », si du moins on veut conserver à ce mot ce que son étymologie a transporté jusqu'à nous, c'est à dire le lien avec la maison (domus). En effet, ce milieu associé, tout à la fois géographique, technique, social, économique, institutionnel, dont dépend la vie de l'animal vivant technicisé d'aujourd'hui, est de plus en plus intégré à des modes d'organisation pratiques trouvant leurs logiques dans le système industriel. Hier, c'était la reproduction de la cellule familiale qui constituait le principe structurant ${ }^{30}$. $S^{\prime}$ il n'y a jamais eu d'animaux domestiques sans milieu associé (même quand lui donnait le nom d'élevage traditionnel), on ne peut plus qualifier de "domestiques" ces animaux, quand les limites du milieu associé dépassent à ce point l'exploitation.

Les expressions convenues "d'animaux de rente" ou "d'animaux de rapports" ne sont pas satisfaisantes non plus. Ces appellations laissent dans l'ombre le poids croissant des dimensions scientifiques et techniques conditionnant les développements les plus récents de ces activités. Voilà pourquoi nous préférons parler "d'êtres vivants technicisés". Depuis que son unité a été proclamée par les biologistes moléculaires, le «vivant » ne connaît pas les frontières entre les règnes. Pour certains des êtres vivants à venir, manipulés génétiquement, il ne sera pas possible de dire s'ils appartiennent au monde des animaux ou des végétaux. Nous avons également préféré le terme " technicisé » à celui « d'artificialisé » puisque, sauf pour les familiers de la pensée simondienne, l'artificiel prend sens dans le système d'opposition avec le "naturel". Or, qui pourrait dire à partir de quand une vache cesse d'être "naturelle" ?

Il nous reste maintenant à répondre à notre troisième question : quel rapport établir entre ce système de production d'êtres vivants technicisés et la prolifération des vaches en images à destination des citadins?

\section{De la vache standardisée à la vache médiatisée}

\footnotetext{
${ }^{29}$ P. 55. Ce milieu associé "techno-géographique" de Gilbert. Simondon n'est pas très différent du milieu "socio-technique" de Michel Callon.

${ }^{30} \mathrm{Tel}$ est le sens de l'article de fond de Catherine et Raphaël Larrère, "Le contrat domestique" (Le Courrier de l'environnement de l'INRA, 30, 1997, pp. 5-17). Voir également les travaux de Jean-Pierre Digard et sa notion de " système domesticatoire européen" (L'homme et les animaux domestiques, anthropologie d'une passion, Paris, Fayard, 1990 et Les Français et leurs animaux, Paris, Fayard, 1999. Ces ouvrages contiennent de précieuses analyses, même si l'évolution du traitement des "animaux de rente" n'est qu'esquissée.
} 
Aujourd'hui, la plupart des vaches laitières ont donc un statut bien particulier, qui fait dépendre leur existence du développement d'un milieu associé toujours plus technicisé. Certes, au plus près de l'animal, c'est toujours l'éleveur qui assure la médiation ultime entre ce milieu et ses lots de vaches. Mais cet éleveur n'est que le dernier maillon du réseau proprement industriel dont l'ensemble forme le système "vache laitière". À l'autre bout de la chaîne se trouve le consommateur de lait, de yaourts ou de fromages, un citadin dont les attaches rurales tendent à disparaître et qui ne sait plus ce qu'est une vache, en dehors de ce que la filière (incluant le marketing) lui en donne à voir par la médiation des vaches en images.

Telle est donc notre thèse : la prolifération des vaches en images est à comprendre comme un effet de la technicisation de l'industrie laitière, non seulement pour faire acheter des produits laitiers, mais pour faire durer le système symbolique sans lequel l'acte de consommation lui-même ne pourrait pas être concevable. Autrement dit, les "vaches imaginées" font partie intégrante de cette filière. Sans elles, les êtres vivants technicisés ne pourraient exister. «Jamais on n'a autant parlé de la vache depuis que la bêtise des hommes l'a rendue folle » peut-on lire ainsi dans un article titré «Vachement sympa » de Version femme ${ }^{31}$.

À l'appui de cette thèse, revenons à l'une de ces premières vaches en image : La vache qui rit. Le "fromage moderne" inventé par Léon Bel est fait d'une pâte homogène au goût moyen réalisé à partir de gruyères, non commercialisables à la coupe à cause de leur défaut d'aspect, fondus et conditionnés en portions individuelles. Il s'agit bien là d'un des tous premiers produits de la société de consommation au sens propre du terme, et d'une des premières campagnes de communication qui ait eu pour support une vache en image. Son premier terrain d'expérimentation fut celui de l'approvisionnement des soldats engagés en masse dans le premier conflit mondial. Depuis, les campagnes de communication se sont multipliées. En 1993, la Cité des Sciences de la Villette organisait une grande exposition intitulée "Le lait, grandeur nature" - une nature qui permet d'euphémiser la technicisation. Le CIDIL était partenaire et initiateur de cette manifestation. Centre d'information au service de la filière professionnelle, cet organisme est en quelque sorte son instrument culturel, chargé de faire la médiation entre nos vaches laitières et le public des urbains consommateurs.

Tout récemment (été 2001), un autre organisme culturel, le CIV (Centre d'Information Viande), lançait une vaste campagne de communication sur les aires de péages des autoroutes des vacances, où était distribué le dépliant "Bovins de France... revue de détail ", assorti de vingt-cinq vignettes autocollantes représentant chacune un spécimen de vache à placer sur une carte de France. Les textes accompagnant le dessin célébraient, comme on pouvait s'y attendre, la diversité du cheptel français « qui ne compte pas moins de 25 races, toutes différentes les unes des autres, tant par leur taille et la couleur de leur robe que par leurs qualités d'élevage et leur aptitude à fournir du lait ou de la viande ». La mise en valeur de la diversité est comme un contrepoint à ce que tout le monde sait : du fait de la spécialisation issue de la sélection génétique, moins de dix races représentent aujourd'hui la quasi-

\footnotetext{
${ }^{31}$ Supplément (numéro 2517, septembre 2001) à nombre de quotidiens régionaux du groupe Hersant..
} 
totalité du cheptel ${ }^{32}$. Pour faire écran à la vache standardisée de l'industrie agroalimentaire, il fallait donc invoquer " la diversité des sols, des climats et des terroirs (qui) a permis à de nombreuses races bovines de développer leurs caractéristiques et de s'intégrer parfaitement dans nos paysages français ». Un autre document beaucoup plus copieux informait le lecteur sur deux sujets d'actualité : l'encéphalite spongiforme bovine et la fièvre aphteuse ${ }^{33}$. Dans ces documents tous les bovins étaient représentés en pleine nature : la vache imaginée semble bien conditionner l'existence de la vache réelle, en contribuant à l'occulter.

À côté de ces campagnes grand public (qui prennent un peu les gens pour des imbéciles), d'autres initiatives se développent depuis peu, qui tendent au contraire à faire connaître aux urbains que nous sommes la réalité de l'élevage bovin. Dominique Dron écrit à ce sujet :

Pour les consommateurs, le hiatus d'image entre les représentations traditionnelles des salons de l'agriculture et du marketing agro-alimentaire, et ce qu'ils découvrent via les médias sur les techniques véritables de production renforce le choc et le rejet suscités par les informations sur la dioxine, les phytosanitaires ou l'ESB 34 .

Certes, le discours reste édulcoré et tout n'est pas montré dans ces «fermes découvertes » et autres opérations portes ouvertes à destination des enfants des écoles ou des adultes désireux d'acheter un fromage de pays. Près de Saint-Etienne, la ferme du Prieuré est une de ces réalisations exemplaires. Depuis une galerie vitrée surplombant la salle de traite, les visiteurs peuvent assister aux opérations avant d'acheter leur fromage, directement du producteur au consommateur. Mais la pédagogie, parfois, n'est pas très éloignée de la propagande. Sur le site de la FNSEA ${ }^{35}$ on peut lire le commentaire suivant, à propos d'une exposition, à Metz, de la Mini Ferme «vitrine du réseau des fermes pédagogiques de Moselle »: "Un parcours initiatique attendait le visiteur. La traçabilité était érigée en leitmotiv avec une mine d'informations sur la composition de l'alimentation des bovins".

Ces initiatives peuvent être rapprochées de toutes celles qui tentent de relancer des productions "traditionnelles" ou de sauver les "races rustiques", fustigées hier pour leur trop faible rentabilité. Est-ce une façon, pour le système productiviste, de reconnaître ses limites, suite à ses récents déboires ? Ces opérations promotionnelles pourraient en être les premiers indices. À ce titre on peut lire dans la livraison d'octobre 2001 de la revue L'amateur de fromage, consacrée à l'époisses, le résumé d'un «ambitieux programme de qualification des élevages», opérationnel depuis janvier 2001. Conditions d'alimentation exceptionnelles (pâturage représentant au moins $50 \%$ de la ration de printemps et fourrage sec représentant $30 \%$ de la ration d'hiver, $85 \%$ de l'alimentation devant provenir de la zone d'appellation), valorisation des races locales (suppression à terme de toutes les races

\footnotetext{
32 En 1994, dans le département de la Loire, deux races (la Prim'Holstein et la Montbéliarde) représentaient $95 \%$ du cheptel des vaches laitières suivies par le Contrôle Laitier (50 \% des vaches du département et $66 \%$ du lait livré).

${ }^{33}$ Voir ces documents sur le site : www.civ-viande.org

34 "Saisir la chance de l'environnement ; les agricultures françaises au milieu du gué", dans Le Courrier de l'environnement de l'INRA, 43, 2001, p. 7.

35 Fédération Nationale des Syndicats d'Exploitants Agricoles.
} 
pies noire «assez étrangère au terroir local» au seul profit des Simmental, Montbéliarde et Brune) : le syndicat fait opérer une "petite révolution » par les éleveurs, qui «ne produisent plus du lait pour du lait, mais du lait pour du fromage ». Symptôme parmi d'autres d'une lente évolution vers une agriculture durable ? Bien difficile à dire tant les structures de la profession, même si les discours changent peu à peu, sont encore fortement orientées par les logiques productivistes.

Mais soyons optimiste : c'est bien des images (et quelles images!) que la dite filière a reçu le plus grand coup en retour, qui pouvait lui faire prendre conscience de sa dérive technicienne. Voilà que les vaches réelles viennent comme faire injure à la représentation sociale qui dit ce que doivent être les vraies vaches (nostalgiques, placides, généreuses, marrantes, psychédéliques ou tout ce qu'on voudra...), mais surtout pas ces carcasses fumantes aux membres raides poussées au bulldozer. Un tout petit détail technique - une économie sur le chauffage des farines animales - a suffi pour que tout l'édifice vacille. Ce n'est pas que beaucoup de vaches soient réellement devenues folles, ni que beaucoup d'humains soient décédés des suites de la maladie de Creutzfeld-Jakob. Mais tout à coup un hiatus est apparu au sein même de la filière agro-alimentaire, entre ce qu'elle était, et l'image qu'elle voulait donner d'elle-même. Et cela d'autant plus fortement qu'il s'agissait d'animaux et d'aliments. $\mathrm{C}^{\prime}$ est à dire de choses dont la nature est d'ordre symbolique autant que d'ordre matériel.

Puisse alors la vache réelle standard, objet technique devenu trop abstrait, céder la place à un être vivant plus concret, c'est-à-dire qui demande une intervention humaine moins artificialisante. On sait aujourd'hui ce qu'il faudrait pour qu'il en soit ainsi : que soit mis en place peu à peu un autre "milieu associé », dans lequel la vache laitière ne serait pas qu'une machine à lait, mais aussi et en même temps un herbivore entretenant les paysages, un auxiliaire animal permettant de répondre à certaines attentes environnementales ${ }^{36}$, un élément du patrimoine "agriculturel » des différentes régions ${ }^{37}$, une espèce dont les différentes races auraient à nouveau droit à l'existence... En bref, un type de vache associé à un mode de «développement durable » qui, selon la définition officielle, « ne viendrait pas compromettre les aspirations des générations futures». On espère bien que ces hommes de demain disposeront toujours de toute sortes de fromages.

\section{Epilogue.}

Nous avons pris le parti de nous en tenir à la vache laitière. Mais peut-on ignorer cette autre articulation majeure entre l'avènement du système industriel de la viande bovine et la production de l'extraordinaire imagerie populaire autour de la figure du garçon vacher («cow-boy» en américain) à la fin du XIXème siècle ? Le système socio-technique d'alors était constitué par le chemin de fer permettant d'expédier les troupeaux vers les abattoirs de Kansas City, d'Omaha et de Chicago et,

\footnotetext{
${ }^{36}$ Conservation des milieux : les références pastorales face aux attentes environnementales, Michel Meuret et François Léger, Projet de recherche Aip Inra "Pâturage" - Rapport final, nov. 2001, INRA.

${ }^{37}$ Voir À propos du patrimoine agriculturel rhônalpin, CREPT Rhône-Alpes, DRAF et DRAC RhôneAlpes, décembre 1998.
} 
de là, vers les centres de consommation des États de l'Est et ceux de l'Europe industrielle. Homme de la frontière (et de l'élevage extensif), le cow-boy incarne alors, et pour longtemps, les valeurs de la conquête et de la liberté... même après que l'introduction progressive du fil de fer barbelé (vers les années 1885) le rende peu à peu inutile. Ainsi en ira-t-il peut-être des vaches imaginées par ces artistes facétieux, si plaisantes à nos yeux, qui continueront longtemps leurs carrières quand on aura oublié le milieu associé agro-industriel qui leur avait donné naissance. 